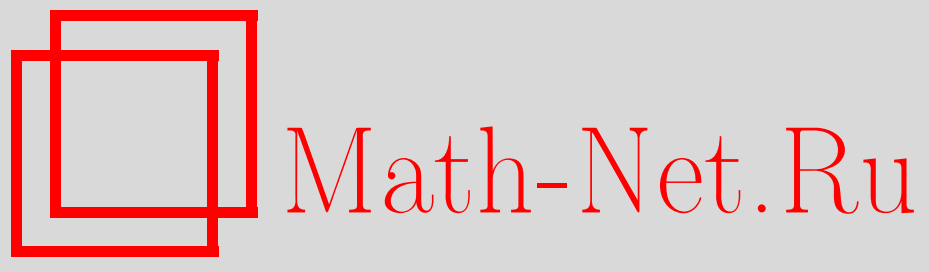

А. В. Силантьев, Функция перехода для цепочки Тоды, TMФ, 2007, том 150, номер 3, 371-390

DOI: https://doi.org/10.4213/tmf5985

Использование Общероссийского математического портала Math-Net.Ru подразумевает, что вы прочитали и согласны с пользовательским соглашением http://www . mathnet.ru/rus/agreement

Параметры загрузки:

IP : 54.80 .73 .141

26 апреля 2023 г., 15:39:39

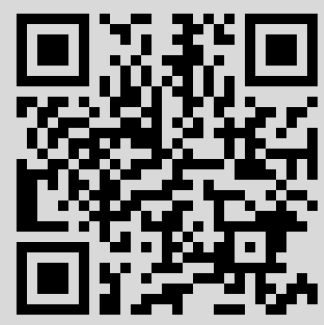




\title{
ФУНКЦИЯ ПЕРЕХОДА ДЛЯ ЦЕПОЧКИ ТОДЫ
}

\begin{abstract}
K задаче нахождения собственных функций открытой цепочки Тоды применен метод $\Lambda$-операторов, развитый Деркачовым, Корчемским и Манашовым. С помощью диаграммной техники, развитой для этих $\Lambda$-операторов, воспроизведена мера Склянина и изучены свойства $\Lambda$-операторов. Данный подход к построению собственных функций открытой цепочки Тоды воспроизводит представление Гаусса-Гивенталя для этих функций.
\end{abstract}

Ключевые слова: цепочка Тоды, разделение переменных, $Q$-операторы.

\section{1. ВВЕДЕНИЕ}

Эта работа была инспирирована статьей [1], посвященной методу разделения переменных для $X X X$-модели. Ключевая идея метода разделения переменных состоит в том, чтобы подобрать такое интегральное преобразование, которое переводит собственные функции квантовых интегралов движения рассматриваемой модели в произведение функций от одной переменной [2]. Если каждая из этих функций удовлетворяет уравнению Бакстера, то исходная функция будет собственной. Ядро, задающее это преобразование, называется функцией перехода. Она может быть построена последовательным применением некоторых операторов $\Lambda_{k}(u)$ : $\Lambda_{N}\left(\gamma_{1}\right) \Lambda_{N-1}\left(\gamma_{2}\right) \cdots \Lambda_{1}\left(\gamma_{N}\right)$. Каждый оператор $\Lambda_{k}(u)$ представляет собой преобразование функций от $k-1$ переменных в функции от $k$ переменных. Свойства функции перехода можно перенести на алгебраические свойства этих операторов (см. раздел 5).

Функция перехода для $N$-частичной периодической цепочки Тоды была найдена в работах [2]-[5]. Там было показано, что в этом случае она пропорциональна собственной функции $(N-1)$-частичной открытой цепочки Тоды с коэффициентом пропорциональности, зависящим от координаты $N$-й частицы. Мы применяем метод, предложенный в работе [1], для получения этих собственных функций в виде произведения $\Lambda$-операторов, воспроизводя тем самым представление ГауссаГивенталя [6], [7] для функций перехода цепочки Тоды.

* Объединенный институт ядерных исследований, Дубна, Московская обл., Россия; Département de Mathématiques, Université d'Angers, 49045 Angers, France.

E-mail: silant@thsun1.jinr.ru, silant@tonton.univ-angers.fr 
Интегральное представление для собственных функций открытой цепочки Тоды, которое получается в описанном ниже подходе, впервые было получено в работе [7] на основе другого метода. Недавно в работе [6] была предложена теоретикогрупповая интерпретация этого представления, которая опирается на разложение Гаусса для группы $G L(N, \mathbb{R})$. Этим оправдано название такого представления функций перехода.

В статье [1] использовался метод триангуляции оператора Лакса [4]. Триангуляция осуществляется калибровочным преобразованием, зависящим от параметров $y_{0}, \ldots, y_{N}$. В периодическом случае налагается условие $y_{0}=y_{N}$, и тогда конструкция, описанная в работе [4], приводит к $Q$-операторам Бакстера [8] для периодической цепочки Тоды. Для построения $\Lambda$-операторов мы налагаем другие граничные условия: $y_{0} \rightarrow-\infty, y_{N} \rightarrow+\infty$, похожие на те, что использованы в работе [1]. Таким образом, $\Lambda$-операторы и $Q$-операторы Бакстера соответствуют различному выбору граничных условий для параметров триангуляции.

Описывая конструкцию собственных функций открытой цепочки Тоды, мы вводим диаграммную технику, подобную той, что развита в работе [1]. Это позволяет свести громоздкие вычисления с ядрами $\Lambda$-операторов к простому манипулированию диаграммами.

Статья построена следующим образом. В разделе 2 мы напоминаем определение рассматриваемой модели, следуя работе [2]. Раздел 3 посвящен описанию собственных функций этой модели как произведений $\Lambda$-операторов и формулировке диаграммной техники [1], необходимой для доказательства основной теоремы, сформулированной и доказанной в этом разделе. В разделе 4 мы используем эту технику, чтобы проверить взаимную ортогональность полученных собственных функций. Как показывают вычисления, нормировка этих функций задается мерой Склянина. Раздел 5 посвящен алгебраическим свойствам $\Lambda$-операторов.

\section{2. ОТКРЫТАЯ ЦЕПОЧКА ТОДЫ}

Квантовая $N$-частичная открытая цепочка Тоды - одномерная модель с экспоненциальным взаимодействием между соседними частицами, которые расположены на прямой. Гамильтониан этой системы определяется равенством

$$
H=\frac{1}{2} \sum_{n=1}^{N} p_{n}^{2}+\sum_{n=1}^{N-1} e^{x_{n}-x_{n+1}},
$$

где $p_{n}=-i \hbar \partial / \partial x_{n}$ - оператор импульса $n$-й частицы. Благодаря трансляционной инвариантности гамильтониана (2.1) полный импульс системы

$$
P=\sum_{n=1}^{N} p_{n}
$$

коммутирует с гамильтонианом, т.е. является интегралом движения. У этой системы имеется $N$ независимых интегралов движения. Их удобно описывать в терминах 
$R$-матричного формализма. Введем $L$-оператор для цепочки Тоды

$$
L_{n}(u)=\left(\begin{array}{cc}
u-p_{n} & e^{-x_{n}} \\
-e^{x_{n}} & 0
\end{array}\right), \quad n=1, \ldots, N,
$$

и матрицу монодромии для $N$-частичной цепочки Тоды

$$
T_{N}(u)=L_{N}(u) \ldots L_{1}(u)=\left(\begin{array}{ll}
A_{N}(u) & B_{N}(u) \\
C_{N}(u) & D_{N}(u)
\end{array}\right),
$$

где $u$ - спектральный параметр. Элементы матрицы монодромии $A_{N}(u), B_{N}(u)$, $C_{N}(u), D_{N}(u)$ суть эрмитовы операторы, полиномиально зависящие от $u$.

Далее нам понадобятся следующие рекуррентные формулы, непосредственно следующие из (2.3) и (2.4):

$$
\begin{aligned}
& A_{N}(u)=\left(u-p_{N}\right) A_{N-1}(u)+e^{-x_{N}} C_{N-1}(u), \\
& C_{N}(u)=-e^{x_{N}} A_{N-1}(u), \\
& A_{N}(u)=\left(u-p_{N}\right) A_{N-1}(u)-e^{x_{N-1}-x_{N}} A_{N-2}(u) .
\end{aligned}
$$

Эти соотношения показывают, что элементы матрицы монодромии $A_{N}(u)$ и $C_{N}(u)$ являются полиномами $u$ степени $N$ и $N-1$, соответственно. Аналогично можно показать, что $B_{N}(u)$ и $D_{N}(u)$ имеют степени $N-1$ и $N-2$.

Матрица монодромии удовлетворяет квантовому $R T T$-соотношению

$$
R(u-v)\left(T_{N}(u) \otimes I\right)\left(I \otimes T_{N}(v)\right)=\left(I \otimes T_{N}(v)\right)\left(T_{N}(u) \otimes I\right) R(u-v)
$$

с рациональной $R$-матрицей

$$
R(u)=I \otimes I+\frac{i \hbar}{u} \mathcal{P}
$$

где $\mathcal{P}$ - матрица перестановки: $\mathcal{P}_{i j, k l}=\delta_{i l} \delta_{j k}$.

Записывая (2.8) поэлементно, получаем $R T T$-соотношения в терминах элементов матрицы монодромии, в частности

$$
A_{N}(u) A_{N}(v)=A_{N}(v) A_{N}(u)
$$

Последнее означает, что $A_{N}(u)$ является производящей функцией интегралов движения для интегрируемой системы с $N$ степенями свободы. Явные вычисления двух первых интегралов показывают, что это не что иное, как интегралы движения для открытой цепочки Тоды:

$$
\begin{gathered}
A_{N}(u)=\sum_{k=0}^{N}(-1)^{k} u^{N-k} H_{k}, \\
H_{0}=1, \quad H_{1}=P, \quad H_{2}=\frac{1}{2} P^{2}-H, \\
{\left[H_{k}, H_{j}\right]=0 .}
\end{gathered}
$$


Вследствие (2.13) существуют общие собственные функции интегралов $H_{k}$, соответствующих собственным значениям $E_{k}$, которые определяются уравнениями

$$
A_{N}(u) \psi_{E}(x)=a_{N}(u ; E) \psi_{E}(x)
$$

где

$$
a_{N}(u ; E)=\sum_{k=0}^{N}(-1)^{k} u^{N-k} E_{k},
$$

$E_{0}=1, E=\left(E_{1}, \ldots, E_{N}\right), x=\left(x_{1}, \ldots, x_{N}\right)$. Записывая собственные значения $E_{k}$ в форме симметрических комбинаций новых вещественных переменных $\gamma=$ $\left(\gamma_{1}, \ldots, \gamma_{N}\right)$ :

$$
E_{k}=\sum_{j_{1}<\cdots<j_{k}} \gamma_{j_{1}} \ldots \gamma_{j_{k}},
$$

можно переписать уравнение (2.14) в следующем виде:

$$
A_{N}(u) \psi_{\gamma}(x)=\prod_{j=1}^{N}\left(u-\gamma_{j}\right) \psi_{\gamma}(x)
$$

Потребуем дополнительно, чтобы решения уравнения (2.16) быстро убывали в области $x_{i} \gg x_{i+1}$. Выбор данного требования для асимптотических свойств решений соответствует тому, что $i$-я частица располагается левее, чем $(i+1)$-я.

\section{3. СОБСТВЕННЫЕ ФУНКЦИИ ДЛЯ ОТКРЫТОЙ ЦЕПОЧКИ ТОДЫ}

В этом разделе будут найдены собственные функции, определенные в предыдущем разделе с помощью уравнения (2.16), которое равносильно системе из $N$ уравнений

$$
A_{N}\left(\gamma_{j}\right) \psi_{\gamma}(x)=0, \quad j=1, \ldots, N .
$$

Собственные значения (2.15) инвариантны относительно перестановок переменных $\gamma_{1}, \ldots, \gamma_{N}$, поэтому будет уместно потребовать вейль-инвариантности собственных функций

$$
\psi_{\sigma(\gamma)}(x)=\psi_{\gamma}(x)
$$

для всех $\sigma \in S_{N}$, где $S_{N}$ обозначает группу перестановок $N$ элементов и $\sigma(\gamma)=$ $\left(\gamma_{\sigma(1)}, \ldots, \gamma_{\sigma(N)}\right)$.

Чтобы решить уравнение (2.16), достаточно найти вейль-инвариантное решение одного из этих уравнений, например, уравнения

$$
A_{N}\left(\gamma_{1}\right) \psi_{\gamma}(x)=0
$$

Для этого рассмотрим следующее калибровочное преобразование матриц Лакса:

$$
\tilde{L}_{n}(u)=M_{n} L_{n}(u) M_{n-1}^{-1}, \quad n=1, \ldots, N,
$$


выполняемое с помощью невырожденных матриц

$$
M_{n}=\left(\begin{array}{cc}
1 & 0 \\
i e^{y_{n}} & 1
\end{array}\right), \quad n=0, \ldots, N .
$$

Тогда деформированная $N$-частичная матрица монодромии примет вид

$$
\widetilde{T}_{N}(u) \equiv\left(\begin{array}{cc}
\tilde{A}_{N}(u) & \widetilde{B}_{N}(u) \\
\widetilde{C}_{N}(u) & \widetilde{D}_{N}(u)
\end{array}\right)=\tilde{L}_{N}(u) \cdots \tilde{L}_{1}(u)=M_{N} T_{N}(u) M_{0}^{-1} .
$$

В частности,

$$
\begin{aligned}
\tilde{L}_{n}(u)_{21} & =i e^{y_{n}}\left(u-p_{n}-i e^{y_{n-1}-x_{n}}+i e^{x_{n}-y_{n}}\right), \\
\widetilde{C}_{N}(u) & =i e^{y_{N}} A_{N}(u)+e^{y_{N}+y_{0}} B_{N}(u)+C_{N}(u)-i e^{y_{0}} D_{N}(u) .
\end{aligned}
$$

Здесь через $\tilde{L}_{n}(u)_{21}$ обозначен нижний недиагональный элемент матрицы $\tilde{L}_{n}(u)$.

Рассмотрим вспомогательное уравнение

$$
\tilde{L}_{n}(u)_{21} w_{n}(u)=0 .
$$

Оно имеет решение

$$
w_{n}(u)=\exp \left(\frac{i}{\hbar} u\left(x_{n}-y_{n-1}\right)-\frac{1}{\hbar} e^{y_{n-1}-x_{n}}-\frac{1}{\hbar} e^{x_{n}-y_{n}}\right) .
$$

Очевидно, что функция

$$
W_{u}(x ; y)=\prod_{n=1}^{N} w_{n}(u)=\exp \sum_{n=1}^{N}\left(\frac{i}{\hbar} u\left(x_{n}-y_{n-1}\right)-\frac{1}{\hbar} e^{y_{n-1}-x_{n}}-\frac{1}{\hbar} e^{x_{n}-y_{n}}\right)
$$

удовлетворяет уравнению

$$
\widetilde{C}_{N}(u) W_{u}(x ; y)=0 .
$$

В пределе $y_{0} \rightarrow-\infty, y_{N} \rightarrow+\infty$ формула (3.8) превращается в равенство

$$
A_{N}(u)=-i \lim _{\substack{y_{0} \rightarrow-\infty \\ y_{N} \rightarrow+\infty}} e^{-y_{N}} \widetilde{C}_{N}(u)
$$

Следовательно, умножая уравнение $(3.12)$ на $-i e^{-y_{N}} e^{(i / \hbar) u\left(y_{0}+y_{N}\right)}$, беря тот же предел, что и в (3.13), и полагая $u=\gamma_{1}$, мы приходим к уравнению (3.3) с решением $\psi_{\gamma}(x)=\Lambda_{\gamma_{1}}(x ; y)$, где

$$
\begin{aligned}
\Lambda_{u}(x ; y) & =\Lambda_{u}\left(x_{1}, \ldots, x_{N} ; y_{1}, \ldots, y_{N-1}\right)=\lim _{\substack{y_{0} \rightarrow-\infty \\
y_{N} \rightarrow+\infty}} e^{(i / \hbar) u\left(y_{0}+y_{N}\right)} W_{u}(x ; y)= \\
& =\exp \left(\frac{i}{\hbar} u\left(\sum_{n=1}^{N} x_{n}-\sum_{n=1}^{N-1} y_{n}\right)-\frac{1}{\hbar} \sum_{n=1}^{N-1}\left(e^{y_{n}-x_{n+1}}+e^{x_{n}-y_{n}}\right)\right) .
\end{aligned}
$$

Обозначим через $\Lambda_{N}(u)$ интегральный оператор с ядром $\Lambda_{u}\left(x_{1}, \ldots, x_{N} ; y_{1}, \ldots\right.$ $\left.\ldots, y_{N-1}\right)$ :

$$
\left(\Lambda_{N}(u) \cdot f\right)(x)=\int_{\mathbb{R}^{N-1}} d y \Lambda_{u}\left(x_{1}, \ldots, x_{N} ; y_{1}, \ldots, y_{N-1}\right) f(y) .
$$


Поскольку модуль ядра $\left|\Lambda_{u}(x ; y)\right|$ быстро убывает при $y_{i} \rightarrow \pm \infty$, интеграл в правой части (3.15) сходится абсолютно для функций $f(y)$, достаточно медленно растущих на бесконечности. Полагая $u=\gamma_{1}$ в (3.15), мы получаем решение уравнения (3.3). Поскольку модуль ядра $\left|\Lambda_{u}(x ; y)\right|$ быстро убывает в области $x_{i} \gg x_{i+1}$, то это решение имеет требуемые асимптотические свойства.

Теорема 1. Решение уравнения (3.3)

$$
\psi_{\gamma}(x)=\left(\Lambda_{N}\left(\gamma_{1}\right) \ldots \Lambda_{2}\left(\gamma_{N-1}\right) \Lambda_{1}\left(\gamma_{N}\right) \cdot 1\right)\left(x_{1}, \ldots, x_{N}\right)
$$

где $\left(\Lambda_{1}\left(\gamma_{N}\right) \cdot 1\right)\left(x_{1}\right)=e^{(i / \hbar) \gamma_{N} x_{1}}$, вейль-инвариантно, т.е. удовлетворяет условию (3.2), и, следовательно, является решением уравнения (2.16), быстро убъвающим в области $x_{i} \gg x_{i+1}$.

ДокАЗАтЕльство. Для доказательства достаточно установить инвариантность относительно элементарных перестановок, т.е. проверить равенство

$$
\begin{aligned}
& \int_{\mathbb{R}^{N-n+1}} d y \Lambda_{\gamma_{n-1}}\left(x_{1}, \ldots, x_{N-n+2} ; y_{1}, \ldots, y_{N-n+1}\right) \Lambda_{\gamma_{n}}\left(y_{1}, \ldots, y_{N-n+1} ; z_{1}, \ldots, z_{N-n}\right)= \\
& =\int_{\mathbb{R}^{N-n+1}} d y \Lambda_{\gamma_{n}}\left(x_{1}, \ldots, x_{N-n+2} ; y_{1}, \ldots, y_{N-n+1}\right) \Lambda_{\gamma_{n-1}}\left(y_{1}, \ldots, y_{N-n+1} ; z_{1}, \ldots, z_{N-n}\right)
\end{aligned}
$$

для $n=2, \ldots, N$.

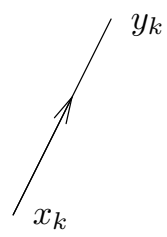

a

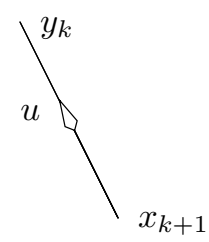

б

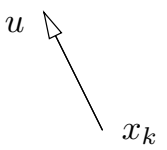

B

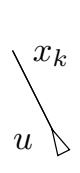

$\Gamma$

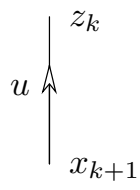

д

Рис. 1

Для этого мы будем использовать метод диаграмм, введенный в работе [1]. На рис. 1 изображены следующие функции:

рис. $1 \mathrm{a}-$

$$
I\left(x_{k}, y_{k}\right)=e^{-(1 / \hbar) e^{x_{k}-y_{k}}},
$$

рис. $16-$

$$
J_{u}\left(x_{k+1}, y_{k}\right)=e^{(i / \hbar) u\left(x_{k+1}-y_{k}\right)-(1 / \hbar) e^{y_{k}-x_{k+1}}},
$$

рис. 1в -

$$
Z_{u}\left(x_{k}\right)=e^{(i / \hbar) u x_{k}},
$$

рис. $1 \Gamma-$

$$
Z_{u}^{-1}\left(x_{k}\right)=e^{-(i / \hbar) u x_{k}},
$$

рис. 1д -

$$
Y_{u}\left(x_{k+1}, z_{k}\right)=\left(1+e^{x_{k+1}-z_{k}}\right)^{(i / \hbar) u} .
$$


Функция (3.16) может быть теперь представлена в графическом виде. Для случая $N=5$ она изображена на рис. 2 , где под точками понимается интегрирование по соответствующим переменным.

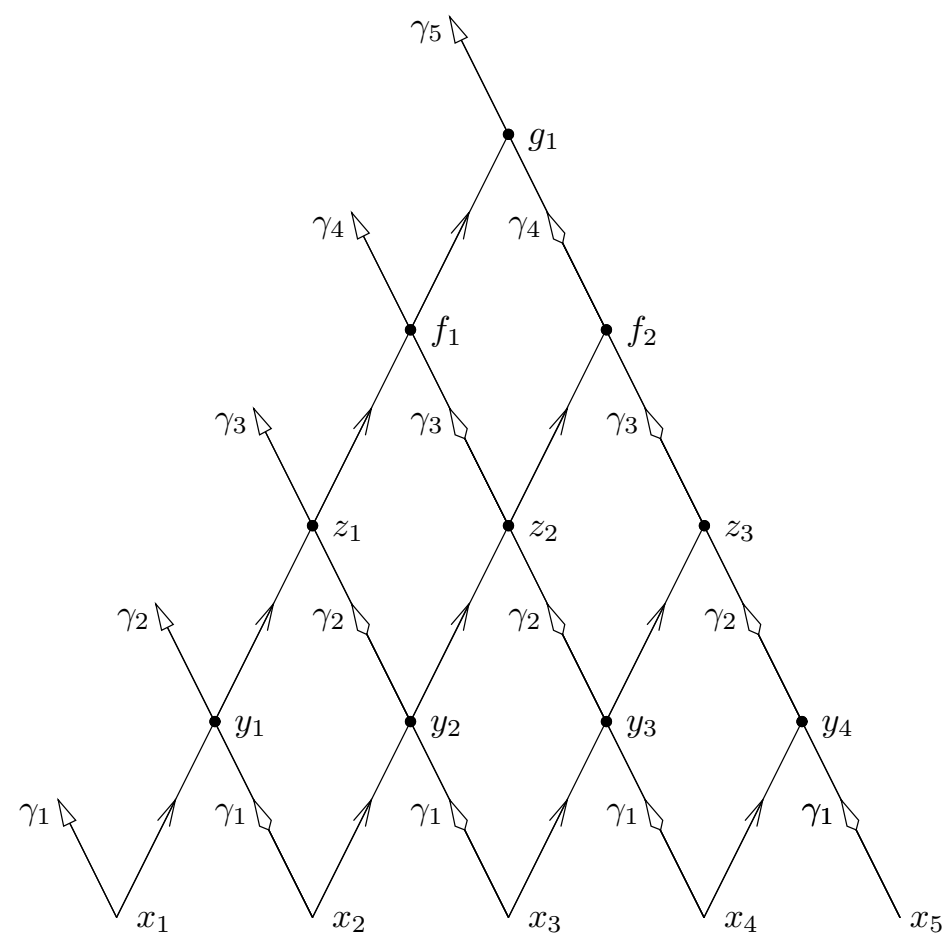

Рис. 2

Лемма 1. Справедливы равенства, изображенные на рис. 3.

ДокАЗАТЕЛЬСтво. Интегрирование по $y_{k}$ в левой части рис. За дает

$$
\begin{array}{rl}
\int_{-\infty}^{+\infty} d y_{k} & I\left(x_{k}, y_{k}\right) J_{\gamma_{n-1}}\left(x_{k+1}, y_{k}\right) I\left(y_{k}, z_{k}\right) J_{\gamma_{n}}\left(y_{k}, z_{k-1}\right)=e^{(i / \hbar)\left(\gamma_{n-1} x_{k+1}-\gamma_{n} z_{k-1}\right)} \times \\
& \times \int_{-\infty}^{+\infty} d y_{k} \exp \left(\frac{i}{\hbar}\left(\gamma_{n}-\gamma_{n-1}\right) y_{k}-\right. \\
& \left.-\frac{1}{\hbar}\left(e^{-x_{k+1}}+e^{-z_{k}}\right) e^{y_{k}}-\frac{1}{\hbar}\left(e^{x_{k}}+e^{z_{k-1}}\right) e^{-y_{k}}\right)= \\
=2 e^{(i / \hbar)\left(\gamma_{n-1} x_{k+1}-\gamma_{n} z_{k-1}\right)}\left(\frac{e^{x_{k}}+e^{z_{k-1}}}{e^{-x_{k+1}+e^{-z_{k}}}}\right)^{\left(i\left(\gamma_{n}-\gamma_{n-1}\right)\right) /(2 \hbar)} \times \\
\quad \times K_{(i / \hbar)\left(\gamma_{n}-\gamma_{n-1}\right)}\left(\frac{2}{\hbar} \sqrt{\left(e^{x_{k}}+e^{z_{k-1}}\right)\left(e^{-x_{k+1}}+e^{-z_{k}}\right)}\right)
\end{array}
$$



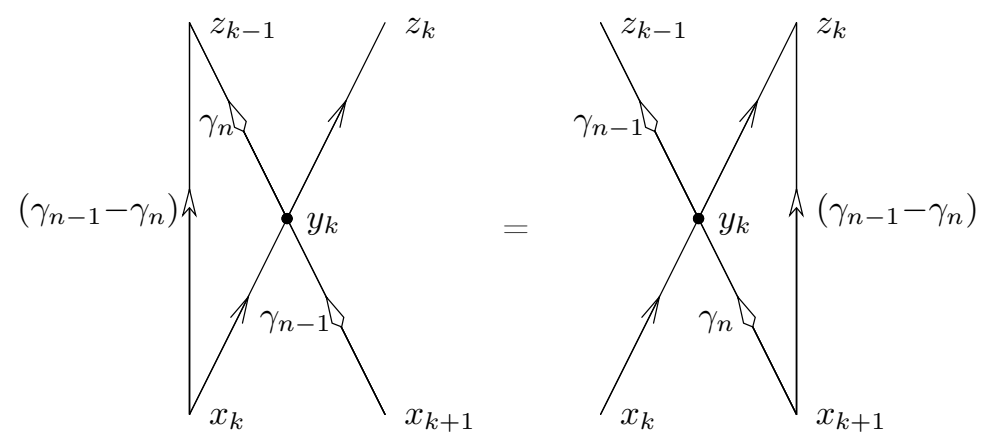

a
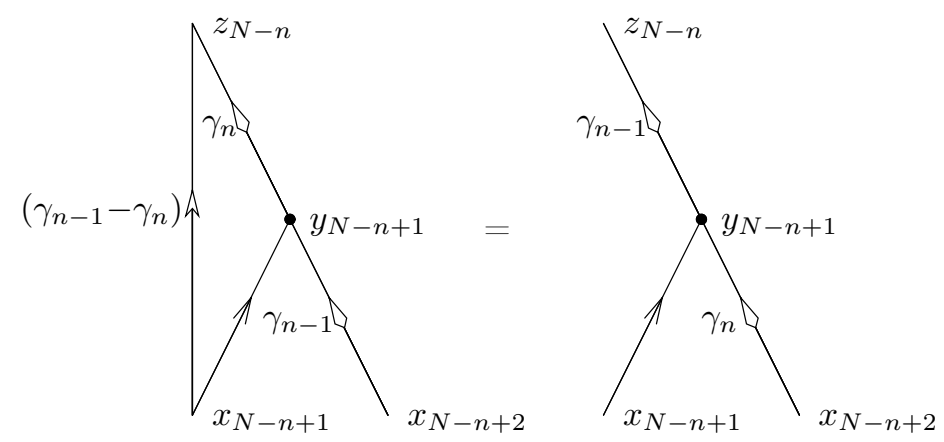

б
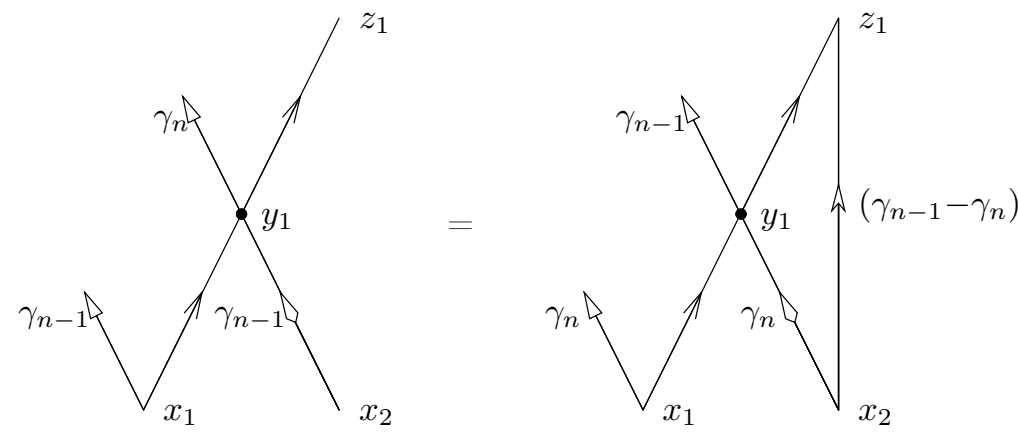

B

Рис. 3 
где $K_{\nu}(z)$ - функция Макдональда [9]. Меняя местами $\gamma_{n-1}$ и $\gamma_{n}$ в (3.18), мы получаем выражение для интеграла в правой части рис. За:

$$
\begin{aligned}
& 2 e^{(i / \hbar)\left(\gamma_{n} x_{k+1}-\gamma_{n-1} z_{k-1}\right)}\left(\frac{e^{x_{k}}+e^{z_{k-1}}}{e^{-x_{k+1}}+e^{-z_{k}}}\right)^{-\left(i\left(\gamma_{n}-\gamma_{n-1}\right)\right) /(2 \hbar)} \times \\
& \times K_{(i / \hbar)\left(\gamma_{n}-\gamma_{n-1}\right)}\left(\frac{2}{\hbar} \sqrt{\left(e^{x_{k}}+e^{z_{k-1}}\right)\left(e^{-x_{k+1}}+e^{-z_{k}}\right)}\right) .
\end{aligned}
$$

Отношение $(3.18)$ к (3.19) в точности равно $Y_{\gamma_{n-1}-\gamma_{n}}\left(x_{k+1}, z_{k}\right) Y_{\gamma_{n-1}-\gamma_{n}}^{-1}\left(x_{k}, z_{k-1}\right)$.

Равенства, изображенные на рис. 3б, в, доказываются аналогично.

Продолжение доказательства теоремы 1 изображено на рис. 4. Здесь показана левая часть равенства (3.17) после применения рис. 3в. Далее, используя рис. За, можно перемещать вертикальную линию, изображающую функцию $Y_{\gamma_{n-1}-\gamma_{n}}\left(x_{j}, z_{j-1}\right)$, направо, как это указано на диаграмме. Когда эта линия достигнет правого края диаграммы, можно применить рис. 3б. В результате $\gamma_{n-1}$ и $\gamma_{n}$ окажутся переставленными, и мы получим правую часть равенства (3.17).

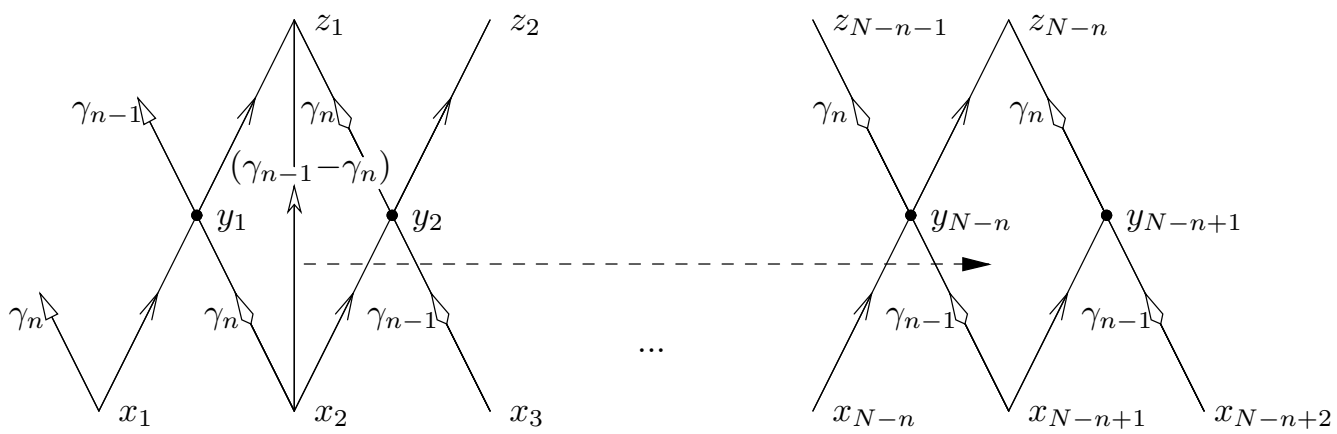

Рис. 4

Подставляя явное выражение (3.14) для ядра одного из операторов вида (3.15) в правую часть равенства (3.16), мы получаем рекуррентную формулу для функции (3.16)

$$
\begin{aligned}
\psi_{\gamma_{1}, \ldots, \gamma_{N}}\left(x_{1}, \ldots, x_{N}\right)=\int_{\mathbb{R}^{N-1}} d y_{1} \ldots d y_{N-1} \psi_{\gamma_{1}, \ldots, \gamma_{N-1}}\left(y_{1}, \ldots, y_{N-1}\right) \times \\
\times \exp \left(\frac{i}{\hbar} \gamma_{N}\left(\sum_{n=1}^{N} x_{n}-\sum_{n=1}^{N-1} y_{n}\right)-\frac{1}{\hbar} \sum_{n=1}^{N-1}\left(e^{y_{n}-x_{n+1}}+e^{x_{n}-y_{n}}\right)\right),
\end{aligned}
$$


которая приводит к следующему интегральному представлению для собственных функций открытой цепочки Тоды:

$$
\begin{aligned}
\psi_{\gamma}\left(z_{N 1}, \ldots, z_{N N}\right) & = \\
= & \int_{\mathbb{R}} \frac{N(N-1)}{2} \prod_{n=1}^{N-1} \prod_{j=1}^{n} d z_{n j} \exp \left(\frac{i}{\hbar}\left[\gamma_{N} \sum_{j=1}^{N} z_{N j}+\sum_{n=1}^{N-1}\left(\gamma_{n}-\gamma_{n+1}\right) \sum_{j=1}^{n} z_{n j}\right]-\right. \\
& \left.\quad-\frac{1}{\hbar} \sum_{n=1}^{N} \sum_{j=1}^{n-1}\left(e^{z_{n j}-z_{n-1, j}}+e^{z_{n-1, j}-z_{n, j+1}}\right)\right)
\end{aligned}
$$

Оно называется представлением Гаусса-Гивенталя [6], [7] для функции перехода в цепочке Тоды.

\section{4. МЕРА ИНТЕГРИРОВАНИЯ}

В этом разделе мы проверим ортогональность полученных собственных функций, используя диаграммную технику. Возникающая при этом нормировочная функция совпадает с мерой Склянина, которая использовалась в методе разделения переменных для периодической цепочки Тоды [2], [3], [10], [11].

ТЕОРЕма 2. Функиия $\psi_{\gamma}(x)$, определяемая формулой (3.16), удовлетворяет условию ортогональности

$$
\int_{\mathbb{R}^{N}} d x \overline{\psi_{\gamma}(x)} \psi_{\gamma^{\prime}}(x)=\mu^{-1}(\gamma) \delta_{\mathrm{SYM}}\left(\gamma^{\prime}, \gamma\right),
$$

где

$$
\delta_{\mathrm{SYM}}\left(\gamma^{\prime}, \gamma\right)=\frac{1}{N !} \sum_{\sigma \in S_{N}} \prod_{i=1}^{N} \delta\left(\gamma_{i}^{\prime}-\gamma_{\sigma(i)}\right)
$$

- симметризованная $\delta$-функция и $\mu(\gamma)$ - мера Склянина:

$$
\mu(\gamma)=\frac{(2 \pi \hbar)^{-N}}{N !} \prod_{k<m}\left[\Gamma\left(\frac{\gamma_{m}-\gamma_{k}}{i \hbar}\right) \Gamma\left(\frac{\gamma_{k}-\gamma_{m}}{i \hbar}\right)\right]^{-1}
$$

Левая часть равенства (4.1) здесь понимается как обобщенная функиия с аргументами $\gamma_{1}^{\prime}, \ldots, \gamma_{N}^{\prime}$, зависящая от попарно различных параметров $\gamma_{1}, \ldots, \gamma_{N}$.

Ввиду эрмитовости операторов $H_{k}$ их собственные функции $\psi_{\gamma}(x)$ образуют полный набор [12]. Это означает, что любая функция $f(x)$, лежащая в гильбертовом пространстве $L^{2}\left(\mathbb{R}^{N}\right)$, может быть представлена в виде интеграла

$$
f(x)=\int_{\mathbb{R}^{N}} \psi_{\gamma}(x) g(\gamma) \mu(\gamma) d \gamma
$$

для некоторой суммируемой функции $g(\gamma)$. Как следствие, мы имеем условие полноты

$$
\int_{\mathbb{R}^{N}} d \gamma \mu(\gamma) \overline{\psi_{\gamma}(x)} \psi_{\gamma}\left(x^{\prime}\right)=\prod_{i=1}^{N} \delta\left(x_{i}-x_{i}^{\prime}\right) .
$$


ДоКАЗАТЕЛЬСТво тЕОРЕмы 2. Прежде всего нам понадобится диаграммное представление функции $\overline{\psi_{\gamma}(x)}$, чтобы вычислить интеграл, стоящий в левой части (4.1), используя диаграммную технику. Для этого рассмотрим диаграмму для $\psi_{\gamma}(x)$, которая изображена на рис. 2 для $N=5$ или на рис. 5а для $N=3$. Выполним следующие шаги.

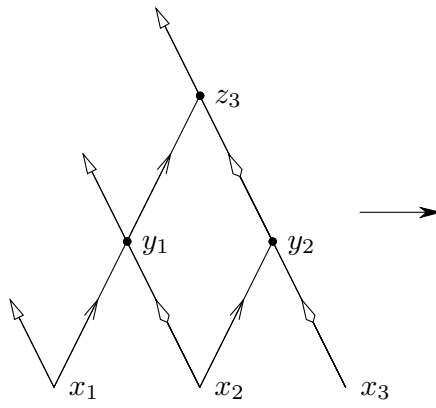

$\mathrm{a}$

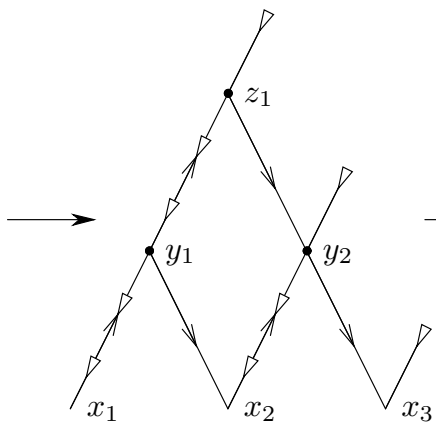

$\Gamma$

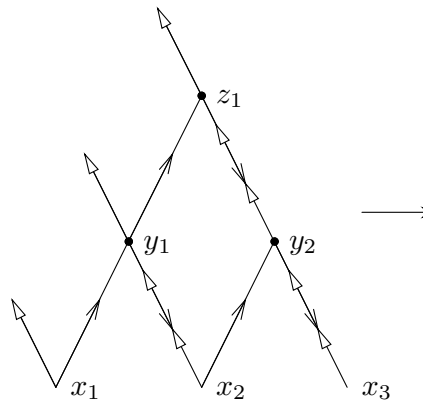

б

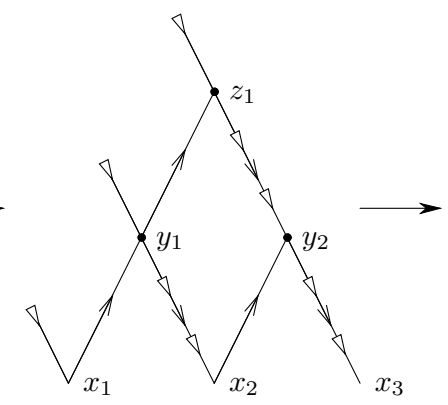

B

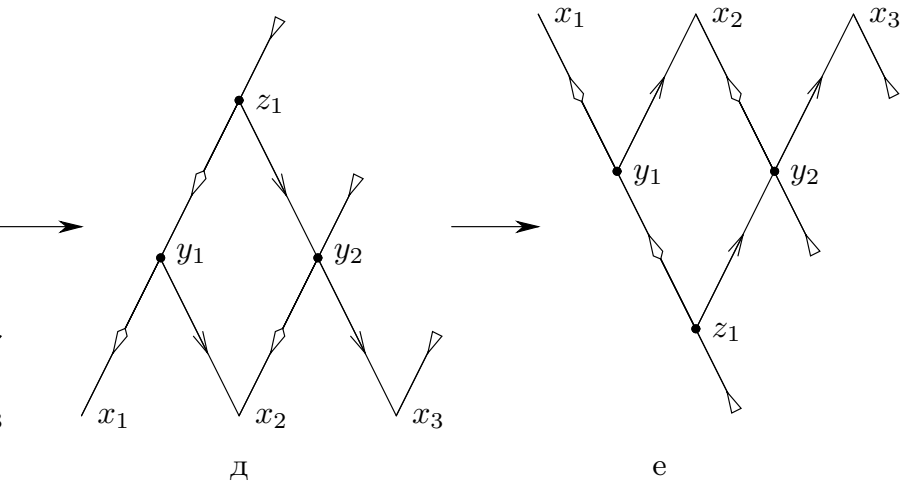

Рис. 5

Шаг 1. Мнимая единица входит лишь в функции $J$ и $Z$. Для того чтобы свести комплексное сопряжение функции $\psi_{\gamma}(x)$ к сопряжению функций $Z$, разложим $J_{u}\left(x_{k+1}, y_{k}\right)$ в произведение $Z_{u}^{-1}\left(y_{k}\right) I\left(y_{k}, x_{k+1}\right) Z_{u}\left(x_{k+1}\right)$ (рис. 6а). Это соответствует переходу от рис. 5 а к рис. $5 б$.

Шаг 2. Заменим все $Z_{u}\left(x_{k}\right)$ на $Z_{u}^{-1}\left(x_{k}\right)$ (рис. 6б) выполняя тем самым комплексное сопряжение. Мы приходим к диаграмме на рис. 5в, которая изображает функцию $\overline{\psi_{\gamma}(x)}$.

Шаг 3. Поскольку линия, изображающая функцию $Z_{u}^{-1}\left(x_{k}\right)$, прикреплена лишь к одной точке (а именно к $x_{k}$ ), можно ее повернуть, как это показано на рис. 6в, г. Диаграммное представление функции $\overline{\psi_{\gamma}(x)}$ после выполнения этого шага представлено на рис. 5г. 


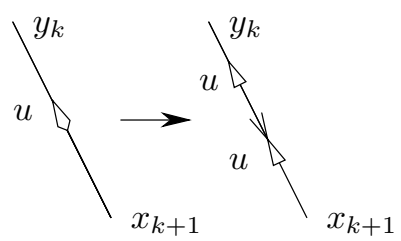

a

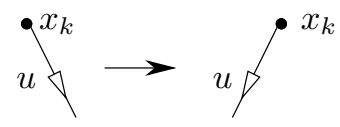

B

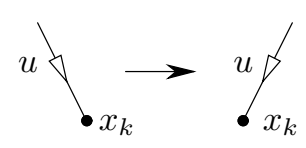

$\Gamma$

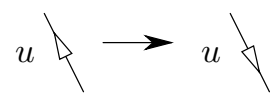

б

Рис. 6
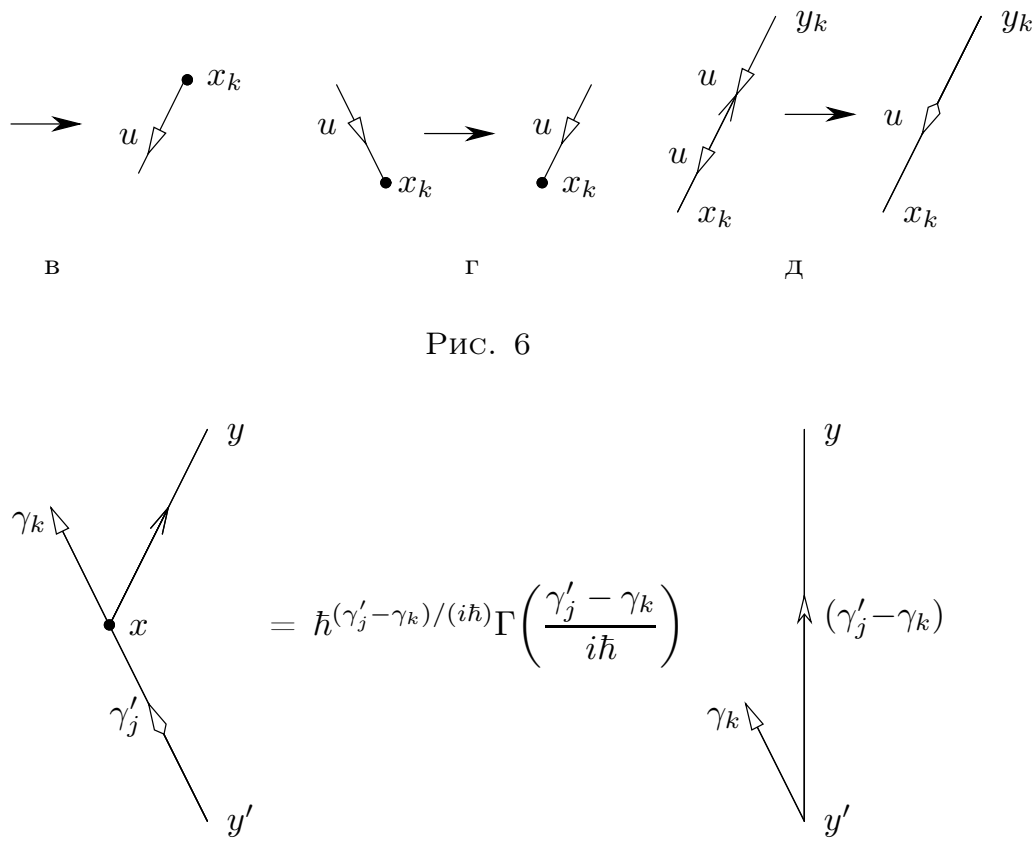

д
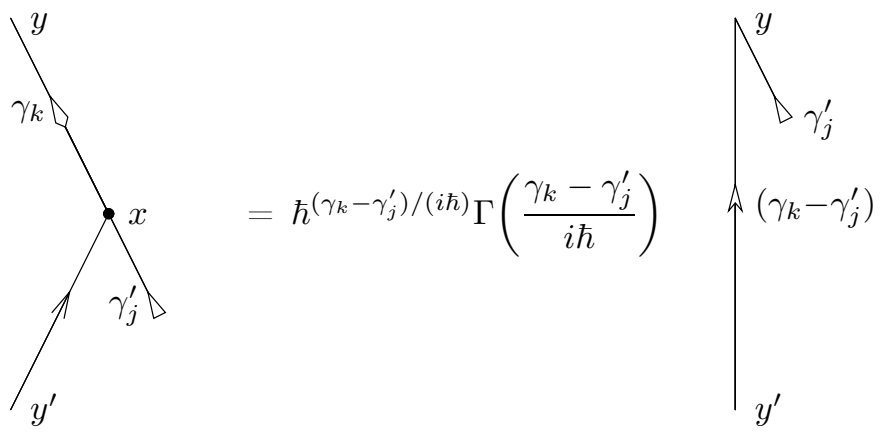

б

Рис. 7 
Шаг 4. Заменим все произведения вида $Z_{u}^{-1}\left(x_{k}\right) I\left(x_{k}, y_{k}\right) Z_{u}\left(y_{k}\right)$ на $J_{u}\left(y_{k}, x_{k}\right)$ (рис. 6д), т.е. получим рис. 5д.

Шаг 5. Наконец, перевернем диаграмму, приведенную на рис. 5д, вверх ногами и получим рис. $5 \mathrm{e}$.

Интеграл в формуле (4.1) соответствует присоединению диаграммы, изображенной рис. 5е для $\overline{\psi_{\gamma}(x)}$, к диаграмме, показанной на рис. 5а для функции $\psi_{\gamma}(x)$, в точках $x_{1}, \ldots, x_{N}$ и интегрированию по этим переменным. Ниже (на рис. 8а) изображено представление для этого интеграла в случае $N=4$. Эту диаграмму можно последовательно упрощать, используя равенства, представленные на рис. 7.

ЛЕмма 2. Если $\gamma_{j}^{\prime} \neq \gamma_{k}$, то справедливы равенства, изображенные на рис. $7 a, б$.

ДокАзАтельство. Диаграммами на рис. 7а, б представлены равенства, которые следуют из интегрального представления для гамма-функций:

$$
\begin{aligned}
\int_{-\infty}^{+\infty} d x Z_{\gamma_{k}}(x) I(x, y) J_{\gamma_{j}^{\prime}}\left(y^{\prime}, x\right) \times & \\
\times & \int_{-\infty}^{+\infty} d x \exp \left(\frac{i}{\hbar}\left[\left(\gamma_{k}-\gamma_{j}^{\prime}\right) x+\gamma_{j}^{\prime} y^{\prime}\right]-\frac{1}{\hbar}\left(e^{x-y}+e^{x-y^{\prime}}\right)\right)= \\
= & e^{(i / \hbar) \gamma_{j}^{\prime} y^{\prime}}\left(\frac{\hbar}{e^{-y}+e^{-y^{\prime}}}\right)^{(i / \hbar)\left(\gamma_{k}-\gamma_{j}^{\prime}\right)} \Gamma\left(\frac{\gamma_{j}^{\prime}-\gamma_{k}}{i \hbar}\right) \hbar^{\left(\gamma_{j}^{\prime}-\gamma_{k}\right) /(i \hbar)} \times \\
& \times \Gamma\left(\frac{\gamma_{j}^{\prime}-\gamma_{k}}{i \hbar}\right) Z_{\gamma_{k}}\left(y^{\prime}\right) Y_{\gamma_{j}^{\prime}-\gamma_{k}}\left(y^{\prime}, y\right), \\
\int_{-\infty}^{+\infty} d x J_{\gamma_{k}}(x, y) I\left(y^{\prime}, x\right) Z_{\gamma_{j}^{\prime}}^{-1}(x) \times & \times \int_{-\infty}^{+\infty} d x \exp \left(\frac{i}{\hbar}\left[\left(\gamma_{k}-\gamma_{j}^{\prime}\right) x-\gamma_{k} y\right]-\frac{1}{\hbar}\left(e^{y-x}+e^{y^{\prime}-x}\right)\right)= \\
= & e^{-(i / \hbar) \gamma_{k} y}\left(\frac{\hbar}{e^{y}+e^{y^{\prime}}}\right){ }^{(i / \hbar)\left(\gamma_{j}^{\prime}-\gamma_{k}\right)} \Gamma\left(\frac{\gamma_{k}-\gamma_{j}^{\prime}}{i \hbar}\right) \hbar^{\left(\gamma_{k}-\gamma_{j}^{\prime}\right) /(i \hbar)} \times \\
& \times \Gamma\left(\frac{\gamma_{k}-\gamma_{j}^{\prime}}{i \hbar}\right) Y_{\gamma_{k}-\gamma_{j}^{\prime}}\left(y^{\prime}, y\right) Z_{\gamma_{j}^{\prime}}(y) .
\end{aligned}
$$

Полюсами гамма-функции являются неположительные целые числа. Поскольку переменные $\gamma_{j}^{\prime}$ и $\gamma_{k}$ не равны и принимают вещественные значения, то правые части рис. $7 \mathrm{a}, б$ корректно определены.

Покажем, что для доказательства теоремы 2 достаточно вычислить интеграл, представленный на рис. 8а, в области значений $\gamma_{i}^{\prime} \neq \gamma_{j}$ при $i+j \neq N+1$. Для этого нам необходимо построить гладкое разбиение единицы, симметричное по $\gamma$, одна из компонент которого равна нулю в некоторой области множества $\gamma_{i}^{\prime}=\gamma_{j}$, $i+j \neq N+1$. Так как переменные $\gamma_{i}$ попарно различны, то существует такое $\epsilon>0$, что $6 \epsilon<\min _{i, j}\left|\gamma_{i}-\gamma_{j}\right|$. Пусть $\eta_{\epsilon}(x)$ - бесконечно дифференцируемая функция одной переменной такая, что $0 \leqslant \eta_{\epsilon}(x) \leqslant 1$ при $x \in \mathbb{R}, \eta_{\epsilon}(x)=1$ при $|x|<\epsilon, \eta_{\epsilon}(x)=0$ при 


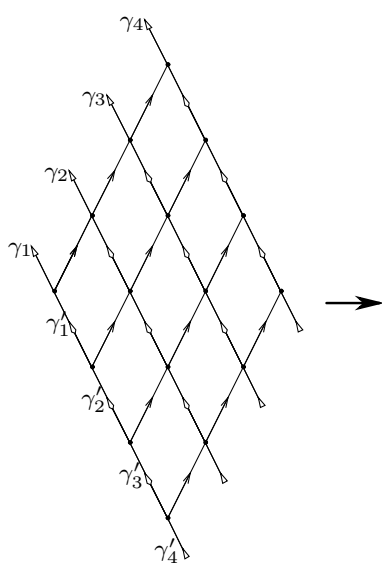

a

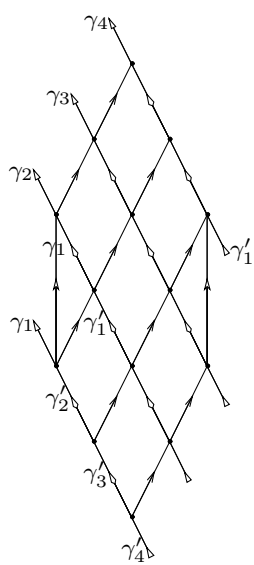

б

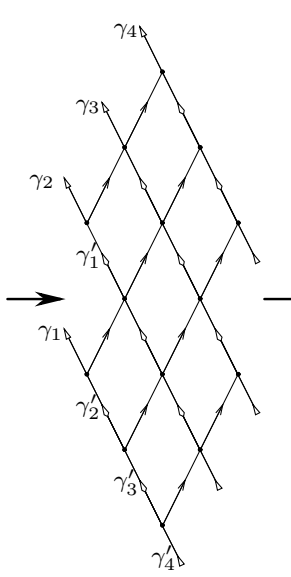

B

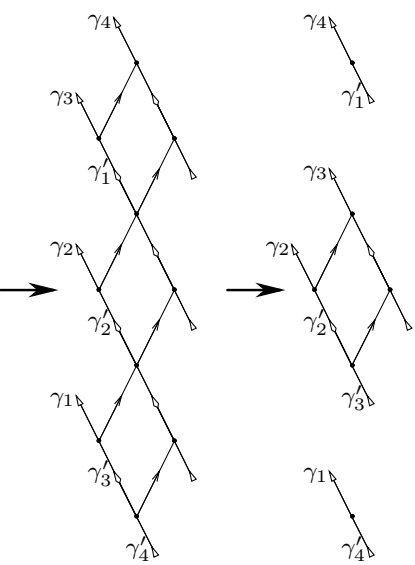

Г

д

Рис. 8

$|x| \geqslant 3 \epsilon$ (см. книгу [13]). Для каждого $\sigma \in S_{N}$ положим

$$
\tilde{\phi}_{\sigma}\left(\gamma^{\prime} ; \gamma\right)=\prod_{\substack{i, j=1 \\ i+j \neq N+1}}^{N}\left(1-\eta_{\epsilon}\left(\gamma_{i}^{\prime}-\gamma_{\sigma(j)}\right)\right) .
$$

Так как по построению функции $\tilde{\phi}_{\sigma}\left(\gamma^{\prime} ; \gamma\right)$ неотрицательны и в любой точке хотя бы одна из них отлична от нуля, то можно осуществить гладкое разбиение единицы функциями

$$
\phi_{\sigma}\left(\gamma^{\prime} ; \gamma\right)=\frac{\tilde{\phi}_{\sigma}\left(\gamma^{\prime} ; \gamma\right)}{\sum_{\sigma^{\prime} \in S_{N}} \tilde{\phi}_{\sigma^{\prime}}\left(\gamma^{\prime} ; \gamma\right)},
$$

имеющими следующие свойства:

1) $\sum_{\sigma \in S_{N}} \phi_{\sigma}\left(\gamma^{\prime} ; \gamma\right)=1$

2) $\phi_{\sigma}\left(\gamma^{\prime} ; \gamma\right)=\phi_{1}\left(\gamma^{\prime} ; \sigma \gamma\right)$, где $\sigma \gamma=\left(\gamma_{\sigma(1)}, \ldots, \gamma_{\sigma(N)}\right)$;

3) если $i+j \neq N+1$, то $\phi_{1}\left(\gamma^{\prime} ; \gamma\right)=0$ при $\left|\gamma_{i}^{\prime}-\gamma_{j}\right|<\epsilon$;

4) $\phi_{1}\left(\gamma^{\prime} ; \gamma\right)=1$ при $\left|\gamma_{i}^{\prime}-\gamma_{N+1-i}\right|<\epsilon$ для всех $i=1, \ldots, N$.

Благодаря свойству 1 искомый интеграл может быть записан в виде

$$
\int_{\mathbb{R}^{N}} d x \overline{\psi_{\gamma}(x)} \psi_{\gamma^{\prime}}(x)=\sum_{\sigma \in S_{N}} \mathcal{I}_{\sigma}\left(\gamma^{\prime} ; \gamma\right),
$$

где

$$
\mathcal{I}_{\sigma}\left(\gamma^{\prime} ; \gamma\right) \phi_{\sigma}\left(\gamma^{\prime} ; \gamma\right)=\int_{\mathbb{R}^{N}} d x \overline{\psi_{\gamma}(x)} \psi_{\gamma^{\prime}}(x) .
$$

Благодаря его симметрии по переменным $\gamma$ и свойству 2, находим

$$
\int_{\mathbb{R}^{N}} d x \overline{\psi_{\gamma}(x)} \psi_{\gamma^{\prime}}(x)=\sum_{\sigma \in S_{N}} \mathcal{I}_{1}\left(\gamma^{\prime} ; \sigma \gamma\right) .
$$


Поэтому для доказательства теоремы достаточно получить равенство

$$
\mathcal{I}_{1}\left(\gamma^{\prime} ; \gamma\right)=\mu^{-1}(\gamma) \frac{1}{N !} \prod_{i=1}^{N} \delta\left(\gamma_{i}^{\prime}-\gamma_{N+1-i}\right)
$$

Свойство 3 означает, что при вычислении $\mathcal{I}_{1}\left(\gamma^{\prime} ; \gamma\right)$ можно использовать лемму 2 при $i+j \neq N+1$, так как полюсы гамма-функций будут зануляться функцией $\phi_{1}\left(\gamma^{\prime} ; \gamma\right)$.

Выражение $\mathcal{I}_{1}\left(\gamma^{\prime} ; \gamma\right)$, которое представляет собой интеграл, показанный на рис. 8a, умноженный на $\phi_{1}\left(\gamma^{\prime} ; \gamma\right)$, может быть вычислено по индукции. Сначала мы последовательно проинтегрируем по граничным точкам диаграммы, связанным с параметрами $\gamma_{1}, \gamma_{1}^{\prime}$. Первое интегрирование ведется по самой левой и самой правой точкам, т.е. по $x_{1}$ и $x_{N}$. Используя рис. 7 , находим рис. 8б. Далее, двигаем вправо вертикальную линию, возникающую в левой части диаграммы (так же как на рис. 4) до тех пор, пока она не сократится с линией, возникающей справа. При этом параметры $\gamma_{1}$ и $\gamma_{1}^{\prime}$ меняются местами. После интегрирования возникает множитель

$$
\Gamma\left(\frac{\gamma_{1}^{\prime}-\gamma_{1}}{i \hbar}\right) \Gamma\left(\frac{\gamma_{1}-\gamma_{1}^{\prime}}{i \hbar}\right)
$$

и мы получаем диаграмму, представленную на рис. 8в, на которой $\gamma_{1}$ и $\gamma_{1}^{\prime}$ связаны уже с другими граничными точками (граничными в смысле рис. 8а). Теперь мы интегрируем по этим точкам. На $k$-м шаге $(k=2, \ldots, N-1) \gamma_{1}$ меняется с $\gamma_{k}^{\prime}$, а $\gamma_{1}^{\prime}$ с $\gamma_{k}$ и появляется множитель

$$
\Gamma\left(\frac{\gamma_{1}^{\prime}-\gamma_{k}}{i \hbar}\right) \Gamma\left(\frac{\gamma_{k}-\gamma_{1}^{\prime}}{i \hbar}\right) \Gamma\left(\frac{\gamma_{k}^{\prime}-\gamma_{1}}{i \hbar}\right) \Gamma\left(\frac{\gamma_{1}-\gamma_{k}^{\prime}}{i \hbar}\right)
$$

На $N$-м шаге имеем множитель

$$
(2 \pi \hbar) \delta\left(\gamma_{1}^{\prime}-\gamma_{N}\right) \cdot(2 \pi \hbar) \delta\left(\gamma_{N}^{\prime}-\gamma_{1}\right)
$$

После этого параметры $\gamma_{1}, \gamma_{1}^{\prime}, \gamma_{N}$ и $\gamma_{N}^{\prime}$ полностью исчезают из диаграммы - они уходят в множители (4.7), (4.8) и (4.9). Оставшаяся диаграмма (средняя часть диаграммы рис. 8д) есть в точности начальная диаграмма, но для $N-2$, которая зависит от параметров $\gamma_{2}, \ldots, \gamma_{N-1}, \gamma_{2}^{\prime}, \ldots, \gamma_{N-1}^{\prime}$. Тем самым получается рекуррентная формула

$$
\begin{aligned}
\phi_{1}\left(\gamma^{\prime} ; \gamma\right) \int & d x_{1} \ldots d x_{N} \overline{\psi_{\gamma_{1}, \ldots, \gamma_{N}}\left(x_{1}, \ldots, x_{N}\right)} \psi_{\gamma_{1}^{\prime}, \ldots, \gamma_{N}^{\prime}}\left(x_{1}, \ldots, x_{N}\right)= \\
=\Gamma & \left(\frac{\gamma_{1}^{\prime}-\gamma_{1}}{i \hbar}\right) \Gamma\left(\frac{\gamma_{1}-\gamma_{1}^{\prime}}{i \hbar}\right) \times \\
& \times \prod_{k=2}^{N-1} \Gamma\left(\frac{\gamma_{1}^{\prime}-\gamma_{k}}{i \hbar}\right) \Gamma\left(\frac{\gamma_{k}-\gamma_{1}^{\prime}}{i \hbar}\right) \Gamma\left(\frac{\gamma_{k}^{\prime}-\gamma_{1}}{i \hbar}\right) \Gamma\left(\frac{\gamma_{1}-\gamma_{k}^{\prime}}{i \hbar}\right) 2 \pi \hbar \delta\left(\gamma_{1}^{\prime}-\gamma_{N}\right) \times \\
& \times 2 \pi \hbar \delta\left(\gamma_{N}^{\prime}-\gamma_{1}\right) \phi_{1}\left(\gamma^{\prime} ; \gamma\right) \times \\
& \times \int d x_{2} \ldots d x_{N-1} \overline{\psi_{\gamma_{2}, \ldots, \gamma_{N-1}}\left(x_{2}, \ldots, x_{N-1}\right)} \psi_{\gamma_{2}^{\prime}, \ldots, \gamma_{N-1}^{\prime}}\left(x_{2}, \ldots, x_{N-1}\right),
\end{aligned}
$$

2 Теоретическая и математическая физика, т. 150, № 3, 2007 г. 
с помощью которой получаем

$$
\mathcal{I}_{1}\left(\gamma^{\prime} ; \gamma\right)=\phi_{1}\left(\gamma^{\prime} ; \gamma\right)=\prod_{\substack{k, j=1 \\ k+j \leqslant N}}^{N-1}\left[\Gamma\left(\frac{\gamma_{j}^{\prime}-\gamma_{k}}{i \hbar}\right) \Gamma\left(\frac{\gamma_{k}-\gamma_{j}^{\prime}}{i \hbar}\right)\right] \prod_{j=1}^{N}(2 \pi \hbar) \delta\left(\gamma_{j}^{\prime}-\gamma_{N+1-j}\right)
$$

Носитель обобщенной функции $\prod_{j=1}^{N}(2 \pi \hbar) \delta\left(\gamma_{j}^{\prime}-\gamma_{N+1-j}\right)$ лежит в области $\mid \gamma_{i}^{\prime}-$ $\gamma_{N+1-i} \mid<\epsilon, i=1, \ldots, N$, в которой $\phi_{1}\left(\gamma^{\prime} ; \gamma\right)=1$. После подстановки $\gamma_{j}^{\prime}=\gamma_{N+1-j}$ в произведение гамма-функций, стоящее в правой части формулы (4.10), учитывая свойство 4 функций $\phi_{1}\left(\gamma^{\prime} ; \gamma\right)$, мы получаем (4.6) с выражением (4.2) для меры Склянина.

\section{5. СВОЙТВА $\Lambda$-ОПЕРАТОРОВ}

$Q$-операторы Бакстера $\widehat{Q}_{N}(u)$ для периодической цепочки Тоды, описанные в работе [4], удовлетворяют следующим свойствам:

а) эти операторы коммутируют для различных значений спектрального параметра: $\left[\widehat{Q}_{N}(u), \widehat{Q}_{N}(v)\right]=0 ;$

б) они коммутируют с трансфер-матрицей $\hat{t}_{N}(u)=A_{N}(u)+D_{N}(u)$ периодической цепочки Тоды: $\left[\widehat{Q}_{N}(u), \hat{t}_{N}(v)\right]=0$;

в) $Q$-операторы удовлетворяют уравнению Бакстера

$$
\hat{t}_{N}(u) \widehat{Q}_{N}(u)=i^{-N} \widehat{Q}_{N}(u-i \hbar)+i^{N} \widehat{Q}_{N}(u+i \hbar) .
$$

В этом разделе мы установим подобные свойства для операторов $\Lambda_{N}(u)$, введенных в разделе 3 .

ПРЕДЛОЖЕНИЕ. $\Lambda$-операторы имеют следующие свойства:

I) $\Lambda_{N}(u) \Lambda_{N-1}(v)=\Lambda_{N}(v) \Lambda_{N-1}(u)$;

II) $A_{N}(u) \Lambda_{N}(v)=(u-v) \Lambda_{N}(v) A_{N-1}(u)$;

III) $C_{N}(u) \Lambda_{N}(u)=i^{-N-1} \Lambda_{N}(u-i \hbar)$;

IV) $B_{N}(u) \Lambda_{N}(u)=i^{N-1} \Lambda_{N}(u+i \hbar)$.

ДокАзАтельство. Свойство I было доказано в теореме 1. Соотношение II было доказано в работе [7] прямым вычислением. Ниже будет приведено упрощенное доказательство этого свойства. Заметим, что оно выполняется на $(N-1)$-частичных собственных функциях $\psi_{\gamma_{1}, \ldots, \gamma_{N-1}}\left(y_{1}, \ldots, y_{N-1}\right)$. В самом деле, благодаря уравнению (2.16) левая часть соотношения II имеет вид

$$
\begin{gathered}
A_{N}(u)\left(\Lambda_{N}(v) \psi_{\gamma_{1}, \ldots, \gamma_{N-1}}\right)\left(x_{1}, \ldots, x_{N}\right)=A_{N}(u) \psi_{\gamma_{1}, \ldots, \gamma_{N-1}, v}\left(x_{1}, \ldots, x_{N}\right)= \\
=(u-v) \prod_{k=1}^{N-1}\left(u-\gamma_{k}\right) \psi_{\gamma_{1}, \ldots, \gamma_{N-1}, v}\left(x_{1}, \ldots, x_{N}\right)
\end{gathered}
$$


при этом правая часть есть

$$
\begin{aligned}
& (u-v)\left(\Lambda_{N}(v) A_{N-1}(u) \psi_{\gamma_{1}, \ldots, \gamma_{N-1}}\right)\left(x_{1}, \ldots, x_{N}\right)= \\
& \quad=(u-v) \prod_{k=1}^{N-1}\left(u-\gamma_{k}\right)\left(\Lambda_{N}(v) \psi_{\gamma_{1}, \ldots, \gamma_{N-1}}\right)\left(x_{1}, \ldots, x_{N}\right)= \\
& =(u-v) \prod_{k=1}^{N-1}\left(u-\gamma_{k}\right) \psi_{\gamma_{1}, \ldots, \gamma_{N-1}, v}\left(x_{1}, \ldots, x_{N}\right) .
\end{aligned}
$$

Сравнивая (5.1) и (5.2), получаем, что равенство II справедливо на функциях $\psi_{\gamma}(x)$. В силу полноты множества этих функций любая функция, принадлежащая области определения операторов $A_{N-1}(u)$ и $\Lambda_{N}(u)$, представима в виде (4.3). Следовательно, свойство II выполняется на всех функциях от $N-1$ переменных, на которых определено действие операторов $A_{N-1}(u)$ и $\Lambda_{N}(v)$.

Для доказательства свойства III нам потребуется следующая формула для действия операторов $A_{m}(u)$ на ядре оператора $\Lambda_{N}(u)$ :

$$
\begin{aligned}
& A_{m}(u) \Lambda_{u}\left(x_{1}, \ldots, x_{N} ; y_{1}, \ldots, y_{N-1}\right)= \\
& \quad=(-i)^{m} \exp \left(\sum_{j=1}^{m}\left(x_{j}-y_{j}\right)\right) \Lambda_{u}\left(x_{1}, \ldots, x_{N} ; y_{1}, \ldots, y_{N-1}\right),
\end{aligned}
$$

где $m=0, \ldots, N-1$ (предполагается, что $\left.A_{0}(u)=1\right)$. Для случаев $m=0$ и $m=1$ эта формула проверяется тривиально. Для $m=2, \ldots, N-1$ она может быть доказана по индукции. В самом деле, используя соотношение (2.7), имеем

$$
\begin{aligned}
A_{m}(u) \Lambda_{u}(x ; y)= & \left(u+i \hbar \partial_{x_{m}}\right) A_{m-1}(u) \Lambda_{u}(x ; y)-e^{x_{m-1}-x_{m}} A_{m-2}(u) \Lambda_{u}(x ; y)= \\
= & {\left[(-i)^{m} \exp \left(\sum_{j=1}^{m-1}\left(x_{j}-y_{j}\right)\right)\left(e^{x_{m}-y_{m}}-e^{y_{m-1}-x_{m}}\right)-\right.} \\
& \left.\quad-(-i)^{m-2} e^{x_{m-1}-x_{m}} \exp \left(\sum_{j=1}^{m-2}\left(x_{j}-y_{j}\right)\right)\right] \Lambda_{u}(x ; y)= \\
= & (-i)^{m} \exp \left(\sum_{j=1}^{m}\left(x_{j}-y_{j}\right)\right) \Lambda_{u}(x ; y) .
\end{aligned}
$$

Формула (5.3) для $m=N-1$ и соотношение (2.6) дают

$$
\begin{aligned}
C_{N}(u) \Lambda_{u}(x ; y) & =-e^{x_{N}} A_{N-1}(u) \Lambda_{u}(x ; y)= \\
& =-(-i)^{N-1} \exp \left(x_{N}+\sum_{j=1}^{N-1}\left(x_{j}-y_{j}\right)\right) \Lambda_{u}(x ; y)=(-i)^{N+1} \Lambda_{u-i \hbar}(x ; y),
\end{aligned}
$$

что в свою очередь означает равенство III.

Заметим, что если положить $m=N$ в (5.4), то член, содержащий $e^{x_{m}-y_{m}}$, не возникает и, следовательно, в правой части мы получаем нуль. Таким образом мы 
доказываем, что ядро $\Lambda_{u}(x ; y)$ удовлетворяет уравнению $(3.1)$, посредством прямого вычисления.

Доказательство свойства IV аналогично. Используя соотношение

$$
B_{N}(u)=\left(u-p_{N}\right) B_{N-1}(u)-e^{x_{N-1}-x_{N}} B_{N-2}(u)
$$

и формулу для действия оператора $B_{m}(u)$ на ядро оператора $\Lambda_{N}(u)$

$$
B_{m}(u) \Lambda_{u}(x ; y)=e^{-x_{1}}(-i)^{m-1} \sum_{k=1}^{m}(-1)^{k+1} \prod_{j=2}^{k} e^{y_{j-1}-x_{j}} \prod_{s=k+1}^{m} e^{x_{s}-y_{s}} \Lambda_{u}(x ; y)
$$

(где $m=0,1, \ldots, N-1)$, которая также доказывается с помощью индукции, получаем

$$
\begin{aligned}
& B_{N}(u) \Lambda_{u}(x ; y)= \\
& =e^{-x_{1}}(-i)^{N-1} \sum_{k=1}^{N-1}(-1)^{k+1} \prod_{j=2}^{k} e^{y_{j-1}-x_{j}} \prod_{s=k+1}^{N-1} e^{x_{s}-y_{s}}\left(-e^{y_{N-1}-x_{N}}\right) \Lambda_{u}(x ; y)- \\
& \quad-e^{-x_{1}}(-i)^{N-3} \sum_{k=1}^{N-2}(-1)^{k+1} \prod_{j=2}^{k} e^{y_{j-1}-x_{j}} \prod_{s=k+1}^{N-2} e^{x_{s}-y_{s}} e^{x_{N-1}-x_{N}} \Lambda_{u}(x ; y)= \\
& =-e^{-x_{1}}(-i)^{N-1}(-1)^{N} \prod_{j=2}^{N} e^{y_{j-1}-x_{j}} \Lambda_{u}(x ; y)=i^{N-1} \Lambda_{u+i \hbar}(x ; y) .
\end{aligned}
$$

Формула (5.6) здесь использована для $m=N-1, N-2$.

Отметим, что с точностью до множителей $(-i)^{\mp N-1}$ свойства III и IV могут быть получены из RTT-соотношения (2.8) и свойства II (см., например, работы [3], [10], [11]). Свойства III и IV ведут к уравнению Бакстера для оператора $\Lambda_{N-1}(u)$. Действительно, учитывая свойство II для $u=v$, получаем

$$
\hat{t}_{N}(u) \Lambda_{N-1}(u)=i^{-N} e^{-x_{N}} \Lambda_{N-1}(u-i \hbar)+i^{N} e^{x_{N}} \Lambda_{N-1}(u+i \hbar) .
$$

\section{6. ЗАКЛЮЧЕНИЕ}

Задача о нахождении собственных функций открытой цепочки Тоды может быть рассмотрена как первый шаг задачи о разделении переменных для периодической цепочки Тоды. Как уже было сказано во введении, разделение переменных достигается специальным выбором функции перехода к новым переменным. В случае цепочки Тоды эта функция должна быть выбрана следующим образом [3]:

$$
U_{\varepsilon, \gamma_{1}, \ldots, \gamma_{N-1}}\left(x_{1}, \ldots, x_{N}\right)=\exp \left(\frac{i}{\hbar}\left(\varepsilon-\sum_{j=1}^{N-1} \gamma_{j}\right) x_{N}\right) \psi_{\gamma_{1}, \ldots, \gamma_{N-1}}\left(x_{1}, \ldots, x_{N-1}\right) \text {. }
$$

Тогда собственные функции интегралов движения периодической цепочки Тоды, определяемых как коэффициенты разложения оператора $\hat{t}_{N}(u)$ по степеням $u$, записываются в виде

$$
\Psi_{E}\left(x_{1}, \ldots, x_{N}\right)=\int_{\mathbb{R}^{N-1}} U_{E_{1}, \gamma_{1}, \ldots, \gamma_{N-1}}\left(x_{1}, \ldots, x_{N}\right) \Phi_{E}\left(\gamma_{1}, \ldots, \gamma_{N-1}\right) \mu(\gamma) d \gamma
$$


Функция (6.1) может быть получена по индукции: имея выражение для функции перехода $(N-1)$-частичной цепочки, можно получить функцию перехода для $N$ частичной. Этот метод был предложен в работе [10], где авторы получают рекуррентную формулу, интегрируя по переменным $\gamma_{j}$ :

$$
\psi_{\lambda_{1}, \ldots, \lambda_{N}}\left(x_{1}, \ldots, x_{N}\right)=\int_{\mathbb{R}^{N-1}} U_{\varepsilon, \gamma_{1}, \ldots, \gamma_{N-1}}\left(x_{1}, \ldots, x_{N}\right) K(\lambda ; \gamma) \mu(\gamma) d \gamma
$$

где $\varepsilon=\sum_{k=1}^{N} \lambda_{k}, \mu(\gamma)$ - мера Склянина, описанная в разделе 4 , а $K(\lambda ; \gamma)$ - некоторое ядро. Эта формула приводит к представлению Меллина-Барнса для функции перехода.

В данной статье показано, что рекуррентное интегрирование может быть реализовано по координатам $x_{n}$ (см. уравнение (3.20)). Это, в свою очередь, ведет к представлению Гивенталя-Гаусса (3.21), полученному ранее в работах [6], [7] из соображений другого сорта. Интегрирование по координатам в формуле (3.20) фактически означает действие оператора $\Lambda_{N}\left(\gamma_{N}\right)$ на $(N-1)$-частичную собственную функцию. Функция (6.1), таким образом, может быть записана через произведение $\Lambda$-операторов, присутствующее в (3.16). Это приводит к тому, что свойства $\Lambda$-операторов, обсуждаемые в разделе 5 , наследуются функцией (6.1). Свойство I, например, означает вейль-инвариантность функции перехода. Благодаря свойствам III и IV справедливо уравнение (5.7), а значит, функция перехода (6.1) удовлетворяет уравнению Бакстера

$$
\hat{t}_{N}\left(\gamma_{k}\right) U_{\varepsilon, \gamma}(x)=i^{-N} U_{\varepsilon, \gamma-i \hbar \delta_{k}}(x)+i^{N} U_{\varepsilon, \gamma+i \hbar \delta_{k}}(x),
$$

где $\delta_{k}=(0, \ldots, 1, \ldots, 0)-k$-й базисный вектор. Благодаря тому, что мера Склянина $\mu(\gamma)$ обладает необходимыми трансляционными свойствами по переменным $\gamma_{i}$, уравнение для собственных значений

$$
\hat{t}_{N}(u) \Psi_{E}(x)=t_{N}(u ; E) \Psi_{E}(x),
$$

где

$$
t_{N}(u ; E)=\sum_{k=0}^{N}(-1)^{k} u^{N-k} E_{k},
$$

эквивалентно тому, что функции $\Phi_{\varepsilon}(\gamma)$ удовлетворяют уравнению Бакстера

$$
t_{N}\left(\gamma_{k} ; E\right) \Phi_{\varepsilon}(\gamma)=i^{N} \Phi_{\varepsilon}\left(\gamma+i \hbar \delta_{k}\right)+i^{-N} \Phi_{\varepsilon}\left(\gamma-i \hbar \delta_{k}\right)
$$

Это, в свою очередь, ведет к возможности представления этих функций в виде

$$
\Phi_{\varepsilon}\left(\gamma_{1}, \ldots, \gamma_{N-1}\right)=\prod_{j=1}^{N-1} c_{\varepsilon}\left(\gamma_{j}\right)
$$

т.е. к разделению переменных (см. работу [3]).

Наличие двух видов рекуррентных формул - типа (6.2) и (3.20) - объясняется тем, что функция $\psi_{\gamma}(x)$ может, с одной стороны, рассматриваться как функция от $x_{n}$, 
удовлетворяющая дифференцальным уравнениям (2.16), а с другой - как функция от $\gamma_{j}$, определяемая некоторой системой разностных уравнений по $\gamma_{j}$ [14], т.е. как волновая фукция некоторой дуальной модели. Эта же дуальность появляется и в теории представлений. Бесконечномерное представление Гельфанда-Цейтлина алгебры Ли $\mathfrak{g l}(N)$ операторами сдвига по переменным $\gamma_{j}$ позволяет получить интегральное представление Меллина-Барнса [15], в то время как представление Гаусса той же алгебры Ли операторами дифференцирования по $x_{n}$ ведет к представлению Гаусса-Гивенталя [6].

Отметим, что метод, предложенный для $X X X$-модели в работе [1] и развиваемый здесь для случая цепочки Тоды (включая использование диаграммной техники), может быть использован для других более сложных интегрируемых систем.

Благодарности. Автор благодарит Лабораторию теоретической физики им. Н. Н. Боголюбова (ОИЯИ) и Department de Mathématiques, Université d'Angers, France за благоприятные условия для работы. Он также признателен Программе франко-русского сотрудничества по теоретической физике, в частности, профессору Ж.-М. Майе за финансовую поддержку данных исследований. Автор выражает свою благодарность С. З. Пакуляку, В. Н. Рубцову и С. М. Харчеву за полезные советы и замечания.

\section{Список литературы}

[1] S. Derkachov, G. Korchemsky, A. Manashov, JHEP, 07 (2003), 047.

[2] E. K. Sklyanin, "The quantum Toda chain", Nonlinear Equations in Classical and Quantum Field Theory, Proc. Semin. (Mendon and Paris, 1983-1984), Lecture Notes in Phys., 226, eds. N. Sanchez, H. DeVega, Springer, Berlin, 1985, 196-233.

[3] S. Kharchev, D. Lebedev, Lett. Math. Phys., 50 (1999), 53-77.

[4] V. Pasquier, M. Gaudin, J. Phys. A, 25 (1992), 5243-5252.

[5] M. Gutzwiller, Ann. Phys., 133 (1981), 304-331.

[6] A. Gerasimov, S. Kharchev, D. Lebedev, S. Oblezin, Int. Math. Res. Notices, 2006 (2006), Article ID 96489; math.RT/0505310.

[7] A. Givental, "Stationary phase integrals, quantum Toda lattices, flag manifolds and the mirror conjecture", Topics in Singularity Theory, Dedicated to V. I. Arnold on the occasion of his 60th birthday, Amer. Math. Soc. Transl., Ser. 2, 180, eds. A. Khovanskii, A. Varchenko, V. Vassiliev, AMS, Providence, RI, 1997, 103-115.

[8] Р. Бэкстер, Точно решаемые модели в статистической механике, Мир, М., 1985.

[9] Г. Бейтмен, А. Эрдейи, Въсшие трансцендентные функиии. Т. 2. Функиии Бесселя, функиии параболического иилиндра, ортогональные многочлены, Наука, М., 1974.

[10] С. М. Харчев, Д. Р.Лебедев, Писъма в ЖЭТФ, 71:6 (2000), 338-343.

[11] S. Kharchev, D. Lebedev, J. Phys. A, 34 (2001), 2247-2258.

[12] И. М. Гельфанд, Н.Я. Виленкин, Обобщенные функции. Bып. 4. Некоторые применения гармонического анализа. Оснащенные гилъбертовы пространства, Физматгиз, M., 1961.

[13] В. С. Владимиров, Обобщенные функиии в математической физике, Наука, М., 1976.

[14] O. Babelon, Lett. Math. Phys., 65:3 (2003), 229-246.

[15] A. Gerasimov, S. Kharchev, D. Lebedev, Int. Math. Res. Not., 17 (2004), 823-854.

Поступила в редакцию 13.03.2006 после доработки 8.07.2006 